\title{
Alterações do TSH em pacientes com sindrome de Down: uma interpretação nem sempre fácil
}

\author{
Alterations of TSH in Down's syndrome patients: a hard interpretation
}

Renato M. Nisihara, MsC'; Shirley R.R. Utiyama, PhD'; Patrícia T. Fiedler, MD²; Nanci P. Oliveira, MD²; Lorete M.S. Kotze, PhD $^{3}$; Iara Messias-Reason, $\mathrm{PhD}^{1}$

\begin{tabular}{|c|c|}
\hline unitermos & resuno \\
\hline $\begin{array}{l}\text { Sindrome de Down } \\
\text { Tireoidites }\end{array}$ & $\begin{array}{l}\text { Objetivo: Determinar as concentrações de hormônio estimulante da tireóide (TSH) e a presença de anticor- } \\
\text { pos antitireoperoxidase (anti-TPO) em pacientes com síndrome de Down (SD) atendidos no ambulatório do } \\
\text { Hospital de Clínicas da Universidade Federal do Paraná. Métodos: Foram incluídos no estudo } 72 \text { pacientes }\end{array}$ \\
\hline TSH & $\begin{array}{l}\text { com SD, não aparentados e selecionados consecutivamente, com média de idade de } 6,15 \text { anos. Oitenta } \\
\text { crianças sas, pareadas com os pacientes, foram utilizadas como controles. Em todas as amostras foram } \\
\text { trica. Resultados: Trinta pacientes com SD }(42,9 \%) \text { apresentaram alterações nas concentrações de TSH, } \\
\text { sendo que } 4,3 \% \text { tinham valores menores que } 0,5 \mu \mathrm{UI} / \mathrm{ml} \text { e } 38,6 \% \text {, valores superiores a } 5 \mu \mathrm{UI} / \mathrm{ml}(5,1 \text { a } \\
22) \text { (média de } 5,56 \pm 4,18 \mu \mathrm{UI} / \mathrm{ml}) \text {. Nos controles, a concentração média de TSH foi } 2,76 \mu \mathrm{UI} / \mathrm{ml}( \pm 1,14) \text {, } \\
\text { evidenciando-se um aumento significativo nos níveis de TSH nos pacientes com SD }(p<0,001) \text {. De forma } \\
\text { similar, caracterizou-se uma diferença significativa na positividade para o anti-TPO nos pacientes }(15,4 \%) \\
\text { em relação aos controles }(0 \% ; p<0,001) \text {. Observou-se ainda aumento significativo nas concentrações de } \\
\text { TSH nos pacientes com idade superior a } 9 \text { anos (média de } 6,86 \pm 4,6 \mu U \mathrm{Ul} / \mathrm{ml}) \text { quando comparados aos } \\
\text { menores de } 9 \text { anos (média de } 5,24 \pm 3,81 \mu \mathrm{UI} / \mathrm{ml} ; p=0,006) \text {, bem como na positividade do anti-TPO } \\
\text { (6/20 vs. } 5 / 52 ; p=0,041) \text {. Conclusões: Os resultados demonstraram alta prevalência de alterações das } \\
\text { dosagens de TSH e de doença tireoidiana nos pacientes com SD, principalmente naqueles com idade } \\
\text { superior a } 9 \text { anos. Os dados indicam que a avaliação da função tireoidiana nos pacientes com SD deve } \\
\text { receber atenção especial dos profissionais de saúde que atendem esses pacientes. }\end{array}$ \\
\hline
\end{tabular}

abstract

Objective: To evaluate the thyroid stimulating hormone (TSH) levels and the presence of antithyroperoxidase antibody (anti-TPO) in Down's syndrome (DS) patients from Hospital de Clínicas of Universidade Federal do Paraná (HC/ UFPR). Methods: Seventy-two DS patients, non-related and consecutively selected (mean age 6.15) were included in the study. Eighty matched healthy children were used as controls. The TSH measurement and the anti-TPO were determined by immunometric assay in all samples. Results: Thirty patients with DS (42.9\%) presented abnormal levels of TSH; $4.3 \%$ showed values below $0.5 \mu \mathrm{IU} / \mathrm{ml}$ and $38.6 \%$ presented values higher than $5 \mu \mathrm{IU} / \mathrm{ml}$ (range 5.1 22; mean $5.56 \pm 4.18 \mu \mathrm{IU} / \mathrm{ml}$ ). The mean concentration of TSH in the controls was $2.76 \pm 1.14 \mu \mathrm{IU} / \mathrm{ml}$, indicating a significant increase in TSH levels in the DS patients $(\mathrm{p}<0.001)$. Similarly, a significant difference was observed in the anti-TPO positivity in the patients' group $(15.4 \%)$ when compared with the controls $(0 \% ; \mathrm{p}<0.001)$. In addition, the TSH levels of patients older than 9 years presented a significant increase (mean of $6.86 \pm 4.6 \mu \mathrm{IJ} / \mathrm{ml}$ ) when compared with the levels observed in patients younger than 9 years (mean of $5.24 \pm 3.81 \mu \mathrm{lU} / \mathrm{ml} ; \mathrm{p}=0.006$ ). The same pattern was observed in the positivity of anti-TPO $(6 / 20$ vs. $5 / 52 ; p=0.041)$. Conclusions: The results demonstrated high prevalence of elevated TSH and anti-TPO in the patients from the DS ambulatory of HC/UFPR, with increased frequency in those older than 9 years. The data indicate that the evaluation of thyroid function in DS patients must receive special attention from health professionals who take care of these patients.

\section{key words}

Down's syndrome

Thyroiditis

TSH

1. Laboratório de Imunopatologia do Hospital de Clínicas da Universidade Federal do Paraná (HC/UFPR).

2. Ambulatório de Síndrome de Down do HC/UFPR.

3. Hospital Universitário Cajuru (HUC) da Pontifícia Universidade Católica do Paraná (PUCPR). 


\section{Introdução}

A síndrome de Down (SD), ou trissomia 21, é o distúrbio cromossômico mais comumente estudado, sendo a causa genética mais importante de retardo mental moderado. Estudos diversos demonstraram que os pacientes com SD possuem inúmeros comprometimentos do sistema imunológico, tais como: diminuição das concentrações séricas de imunoglobulinas, alteração na maturação de linfócitos, disfunção na quimiotaxia de neutrófilos, deficiência parcial de proteínas do sistema complemento e baixas concentrações plasmáticas de zinco, entre outras. Esses defeitos acarretam aos pacientes maior tendência ao desenvolvimento de infecções de repetição e doenças auto-imunes ${ }^{(7,10)}$. Alguns estudos sugerem que uma atividade deficiente do timo estaria implicada nessa característica dos pacientes com SD ${ }^{(6)}$, além da senescência precoce do sistema imune observada nesses indivíduos ${ }^{(1)}$.

A tireoidite auto-imune é a associação mais freqüente de doença auto-imune com SD. Os sintomas e sinais clínicos de hipotireoidismo, principalmente se tiverem início gradual, correm o risco de não serem facilmente identificados nos pacientes com SD, já que podem ser atribuídos à própria síndrome. Portanto, a utilização de parâmetros laboratoriais que avaliem a função tireoidiana é altamente recomendada no acompanhamento desses pacientes. Vários estudos descrevem disfunções tireoidianas nos pacientes com SD, sendo a diminuição da produção dos hormônios da tireóide e a dosagem sérica elevada de hormônio estimulante da tireóide (TSH) os achados mais freqüentes ${ }^{(2,9)}$. Além disso, auto-anticorpos dirigidos contra a tireóide são encontrados entre $13 \%$ e $34 \%$ dos pacientes $(2,5,11)$. O diagnóstico precoce e correto das doenças da tireóide proporciona benefícios diretos tanto no desenvolvimento físico como no intelectual de crianças com SD.

A dosagem de TSH deve ser protocolo em todos os serviços que atendem os portadores de SD. No entanto, na literatura médica há muita controvérsia em relação aos valores normais para os pacientes com SD, uma vez que muitos deles apresentam TSH aumentado e não evidenciam alterações clínicas compatíveis com hipotireoidismo. A dúvida que permanece é se deve ser instituída ou não a reposição hormonal.

O presente estudo teve como objetivo investigar a prevalência de alterações das dosagens de TSH e positividade de anticorpos antitireoperoxidase (anti-TPO) em pacientes portadores de SD atendidos no ambulatório do Hospital de Clínicas da Universidade Federal do Paraná (HC/UFPR).

\section{Materiais e métodos}

O presente estudo foi submetido e aprovado pelo Comitê de Ética em Pesquisas do HC/UFPR.

Foram estudados 72 pacientes atendidos no ambulatório de SD do HC/UFPR, não aparentados e consecutivamente selecionados, sendo $54,1 \%(39 / 72)$ do sexo masculino e $45,9 \%(33 / 72)$ do sexo feminino. A faixa etária variou de 2 a 18 anos, com média de idade de 6,15 anos.

Como grupo controle foram utilizados 80 crianças e adolescentes sadios, provenientes da mesma área geográfica dos pacientes, atendidos rotineiramente em uma Unidade de Saúde da Prefeitura Municipal de Curitiba, Paraná. Eles foram pareados com o grupo de pacientes em estudo, sendo $51,2 \%$ (41/72) do sexo masculino e $48,8 \%$ (39/72) do sexo feminino, com média de idade de 8,02 anos, variando entre 2 e 18 anos.

Foram coletados de cada paciente e controle cerca de $3 \mathrm{ml}$ de sangue sem anticoagulante. Após centrifugação e separação, as alíquotas de soro foram imediatamente armazenadas a $-80^{\circ} \mathrm{C}$ até a sua utilização.

\section{Pesquisa do anticorpo antitireoperoxidase}

A pesquisa de anti-TPO foi realizada empregando-se 0 método de dosagem imunométrica (DPC/Medlab, EUA), em conjunto com o analisador automatizado Immulite, de acordo com as instruções do fabricante. As amostras dos pacientes e dos controles foram diluídas a 1:100 em tampão próprio e processadas pelo equipamento, sendo os dados comparados com os calibradores que acompanham o sistema comercial. Foram consideradas negativas as amostras com valores inferiores a $35 \mathrm{UI} / \mathrm{ml}$.

\section{Dosagem de hormônio estimulante da tireóide}

Foi realizada através do método de dosagem imunométrica (DPC/Medlab, EUA), que utiliza o sistema automatizado Immulite, seguindo as instruções do fabricante. As amostras dos pacientes e dos controles foram testadas sem diluição e analisadas de acordo com a curva estabelecida por calibradores fornecidos pelo kit. De acordo com o fabricante, os valores de TSH tidos como referência para a população saudável se situam entre $0.5 \mu \mathrm{Ul} / \mathrm{ml}$ e $5 \mu \mathrm{UI} / \mathrm{ml}$.

\section{Análise estatística}

Para análise dos dados foram empregados os testes não-paramétricos Mann-Whitney e do qui-quadrado, utilizando-se o programa Microsoft Statistica 5.0. 


\section{Resultados}

Os resultados obtidos estão resumidos nas Tabelas 1

e 2. Trinta pacientes com SD (30/72, $42,9 \%), 16$ do sexo feminino e 14 do masculino, apresentaram alterações nas dosagens de TSH. Deles, 4,3\% (3/72) apresentaram valores inferiores a $0,5 \mu \mathrm{Ul} / \mathrm{ml}$, e $38,6 \%$ (27/72) evidenciaram valores superiores a $5 \mu \mathrm{UI} / \mathrm{ml}(5,3-22,1 \mu \mathrm{Ul} / \mathrm{ml}$, média de 5,56 \pm $4,18 \mu \mathrm{Ul} / \mathrm{ml})$, sendo que sete $(9,7 \%)$ deles apresentavam TSH superior a $10 \mu \mathrm{UI} / \mathrm{ml}$ e $20(27,7 \%)$, dosagens entre 5 e $9,5 \mu \mathrm{Ul} / \mathrm{ml}$. A revisão dos prontuários indicou que os pacientes apresentando TSH suprimido $(n=3)$ na época do estudo já faziam tratamento com reposição hormonal. A dosagem de TSH efetuada no grupo controle demonstrou média de $2,76 \pm 1,14 \mu \mathrm{UI} / \mathrm{ml}$, variando entre 0,64 e $4,90 \mu \mathrm{UI} / \mathrm{ml}$. Esses dados evidenciaram aumento significativo $(p<0,001)$ nas concentrações de TSH nos pacientes com SD.

Onze pacientes $(11 / 72,15,4 \%)$ foram positivos para o anticorpo anti-TPO, sendo sete do sexo feminino e quatro do masculino. Suas concentrações do anti-TPO variaram entre 36 e $1.000 \mathrm{Ul} / \mathrm{ml}$. Nenhum indivíduo do grupo controle foi positivo para anti-TPO, caracterizando uma diferença significativa em relação aos pacientes com SD $(p<0,001)$.

No tocante à idade, observou-se aumento significativo nas concentrações de TSH nos pacientes com SD e idade superior a 9 anos (média de 6,86 $\pm 4,6 \mu \mathrm{Ul} / \mathrm{ml}$ ) quando comparados aos menores de 9 anos (média de 5,24 \pm $3,81 \mu \mathrm{Ul} / \mathrm{ml} ; p=0,006$ ). De forma similar, a positividade do anti-TPO também foi significativamente maior nas crianças acima de 9 anos $(30 \%, 6 / 20)$ em relação às de menor idade $(9,6 \%, 5 / 52 ; p=0,041)$.

Não foi evidenciada diferença significativa nas dosagens de TSH e na positividade do anti-TPO em relação ao sexo dos pacientes com SD.

\section{Discussão}

Nos últimos anos, os avanços da medicina no acompanhamento e no tratamento dos portadores de SD possibilitaram aumento significativo na expectativa de vida e melhora na saúde global desses pacientes, devido principalmente à prevenção das complicações relacionadas à SD. Além dos principais sinais clínicos, como retardo mental, doença cardíaca congênita e hipotonia muscular, os indivíduos com SD apresentam importantes alterações no sistema imunológico que podem implicar em maior predisposição a doenças auto-imunes e infecções recorrentes. Entre as doenças autoimunes, a mais comum na SD é a que afeta a tireóide.

No presente estudo foi observado que 42,9\% (30/72) dos pacientes com SD atendidos no ambulatório do $\mathrm{HC} /$ UFPR apresentavam alterações nas dosagens de TSH. Com relação aos anticorpos anti-TPO, observou-se que 15,4\% $(11 / 72)$ dos pacientes com SD eram positivos para esse marcador, configurando um perfil laboratorial de tireoidite auto-imune. Os resultados obtidos estão de acordo com os descritos por outros autores que também demonstraram essas alterações ${ }^{(5,9,11)}$. Tuysuz e Beker ${ }^{(14)}$, na Turquia, investigaram a função tireoidiana em 320 crianças com SD, sendo

Tabela 1

Concentrações séricas de TSH e positividade do anticorpo antitireoperoxidase nos grupos

\begin{tabular}{lccc}
\hline & Pacientes com SD $(n=72)$ & Controles sadios $(n=80)$ & $p$ \\
TSH $\mu \mathrm{UU} / \mathrm{ml}$ (média $\pm \mathrm{dpm})$ & $5,56 \pm 4,18$ & $2,76 \pm 1,14$ & $<0,001^{*}$ \\
Anti-TPO positivos & $15,4 \%$ & $0 \%$ & $<0,001^{* *}$ \\
\hline
\end{tabular}

*Teste de Mann-Whitney; **teste do qui-quadrado; SD: síndrome de Down; dpm: desvio padrão da média.

Tabela 2

Concentrações séricas de TSH e positividade de anticorpo antitireoperoxidase de acordo com a

\begin{tabular}{lccc}
\hline & Pacientes com idade $>9$ anos & Pacientes com idade $<9$ anos & $p$ \\
& $(n=20)$ & $(n=52)$ & \\
TSH $\mu \mathrm{UI} / \mathrm{ml}($ média $\pm \mathrm{dpm})$ & $6,86 \pm 4,6$ & $5,24 \pm 3,81$ & $0,006^{*}$ \\
Anti-TP0 positivos & $30 \%$ & $9,6 \%$ & $0,041^{* *}$ \\
\hline
\end{tabular}

*Teste de Mann-Whitney; **teste do qui-quadrado; SD: síndrome de Down; dpm: desvio padrão da média. 
que $25,3 \%$ apresentavam hipotireoidismo com aumento


tinham TSH entre 6 e $10 \mu \mathrm{UI} / \mathrm{ml}$, totalizando assim 34,4\% dos pacientes com níveis aumentados de TSH. Zori et al. ${ }^{(15)}$, nos Estados Unidos, evidenciaram disfunção tireoidiana em $66 \%(41 / 61)$ dos pacientes com SD, com valores de TSH acima de $5 \mu \mathrm{UI} / \mathrm{ml}$. Anticorpos antitireóide foram positivos em 28\% (17/61) dos pacientes nesse estudo.

Alguns autores têm demonstrado que a presença de anticorpos antitireóide está relacionada à idade dos pacientes com SD. Karlsson et al.(4) investigaram 85 crianças com SD e verificaram que $35,3 \%$ delas apresentavam disfunção tireoidiana, sendo que apenas uma tinha anti-TPO positivo. Os autores observaram que o hipotireoidismo estava presente em um terço dos pacientes com idade inferior a 25 anos e que a tireoidite auto-imune foi menos freqüente em crianças menores de 5 anos, prevalecendo após os 8 anos de idade. Os resultados do presente estudo corroboram esses achados ao caracterizar diferença significativa nas dosagens de TSH nos pacientes com idade acima de 9 anos quando comparados com os menores de 9 anos ( $p=$ $0,0006)$. Ressalta-se ainda a maior positividade do anti-TPO $(p=0,041)$ nessa faixa etária ( $>9$ anos).

Em relação ao sexo, não foi observada diferença significativa nas dosagens de TSH e na positividade de anti-TPO no grupo de pacientes com SD. Alguns autores demonstraram que na SD, ao contrário da população geral em que as mulheres são mais afetadas pelas doenças tireoidianas, não houve diferenças na prevalência de anticorpos antitireóide quando se compararam os sexos os pacientes ${ }^{(9,13)}$.

Dezenove pacientes com SD $(26,4 \%, 19 / 72)$ apresentaram aumento de TSH e anticorpo anti-TPO negativo. A conduta recomendada nesses casos é que seja instituída a terapêutica hormonal nos pacientes que apresentaram TSH superior a $10 \mu \mathrm{Ul} / \mathrm{ml}$. Nos demais pacientes, que apresentam TSH entre 5 e 10 $1 \mathrm{UI} / \mathrm{ml}$, deve ser continuada a avaliação clinicolaboratorial a fim de se averiguar se ocorre evolução para doença tireoidiana ${ }^{(2,4)}$.

Considerando-se que a deficiência de hormônios da tireóide acarreta prejuízos no desenvolvimento físico e mental dos pacientes, o tratamento deve ser instituído nos casos preconizados. Alguns autores sugerem que a terapêutica hormonal não deve ser utilizada nos casos de hipotireoidismo subclínico sem auto-anticorpos positivos e que esses pacientes requerem observação criteriosa, com acompanhamento clínico e laboratorial trimestral. Por outro lado, o grupo com hipotireoidismo subclínico e anticorpo antitireóide positivo deve ter instituída a terapêutica hormonal apropriada, uma vez que com freqüência esses pacientes evoluem para um quadro de disfunção tireoidiana definida ${ }^{(2,11)}$. Selikowitz ${ }^{(12)}$ acompanhou durante cinco anos um grupo de 101 crianças com SD, sendo que nesse período dez delas desenvolveram hipotireoidismo. $\mathrm{O}$ autor concluiu que não houve diferença significativa no crescimento e no desenvolvimento das crianças com SD e hipotireoidismo quando comparadas aos pacientes com SD sem tal disfunção. $\mathrm{O}$ autor sugere ainda que uma grande proporção das disfunções de tireóide das crianças com SD seja transitória e que o aumento de TSH nesses indivíduos possa ser devido à secreção inapropriada de TSH ou à reação de insensibilidade da tireóide ao TSH, não estando necessariamente relacionado à tireoidite auto-imune. Estudo recente de Prasher e Haque ${ }^{(8)}$ verificando a função tireoidiana em 110 pacientes com SD no Reino Unido demonstrou que cerca de dois terços deles apresentaram disfunção tireoidiana, apesar de serem clinicamente eutireóideos. Esses autores também sugerem que é necessária uma revalidação dos valores ditos normais para os parâmetros laboratoriais quando os mesmos são aplicados à população com SD. Além disso, questionam que possivelmente pacientes com SD possam estar sendo inapropriadamente tratados para uma desordem médica inexistente ${ }^{(8)}$. Em outro trabalho, Gibson et al. realizaram um estudo longitudinal da função tireoidiana em pacientes com SD nas primeiras duas décadas de vida e observaram que podem ocorrer alterações transitórias dos níveis de TSH e ainda que a presença de auto-anticorpos infere alto valor preditivo na evolução para hipotireoidismo ${ }^{(3)}$.

O tratamento dos pacientes com hipotireoidismo subclínico, no entanto, ainda é controverso, necessitando que um maior número de estudos prospectivos seja realizado a fim de estabelecer um protocolo de intervenção terapêutica adequado. Fica evidenciado também que a interpretação de TSH aumentado depende de criteriosa observação clínica do paciente com SD e que há muita controvérsia na literatura.

Finalizando, os resultados obtidos no presente estudo demonstraram a alta prevalência de alterações nos exames de tireóide nos pacientes com SD atendidos no ambulatório de SD do HC/UFPR e corroboram os achados já descritos em outras populações. Adicionalmente, sugere-se que a avaliação da função tireoidiana nos pacientes com SD deva receber atenção especial dos profissionais de saúde que atendem esses pacientes.

\section{Agradecimentos}

Apoio financeiro da Fundação Araucária do Paraná. 


\section{Referências}

I. CUADRADO, E.; BARRENA, M.J. Immune dysfunction in Down's syndrome: primary immune deficiency or early senescence of the immune system? Clin Immunol Immunopathol, v. 78, p. 209-14, 1996.

2. DINANI, S.; CARPENTER, S. Down syndrome and thyroid disorder.J Ment Def Res, v. 34, p. 187-93, 1990.

3. GIBSON, P. A. et al. Longitudinal study of thyroid function in Down's syndrome in the first two decades. Arch Dis Child, v. 90, p. 574-8, 2005.

4. KARLSSON, B. et al. Thyroid dysfunction in Down's syndrome: relation to age and thyroid autoimmunity. Arch Dis Child, v. 79, p. 242-5, 1998.

5. LOUDON, M. M.; DAY, R. E.; DUKE, E. M.Thyroid disease in Down syndrome. Arch Dis Child, v. 60, p. I |49-5।, 1985.

6. MURPHY, M.; EPSTEIN, L. B. Down syndrome peripheral blood contains phenotypically mature CD3 TCR alpha and beta cells but abnormal proportions of TCR gamma delta, TCR alpha beta and CD 4+ 45 RA cells: evidence for an inefficient release of matureT cells by DS thymus. Clin Immun Immunopathol, v. 62, p. 245-5I, 1992.

7. NESPOLI, L. et al. Immunological features of Down's syndrome: a review.J Intell Disab Res, v. 37, n. 6, p. 543-5I, 1993.
8. PRASHER, V:; HAQUE, M. S. Misdiagnosis of thyroid disorders in Down syndrome: time to re-examine the myth? Am J Ment Retard, v. I I0, n. I, p. 23-7, 2005.

9. PUESCHEL, S. M.; PEZZULO, J. C. Thyroid dysfunction in Down syndrome. Am J Dis Child, v. I39, p. 636-9, 1985.

10. RIBEIRO, L. M. A. et al. Avaliação dos fatores associados a infecções recorrentes e/ou graves em pacientes com síndrome de Down.J Pediatr, v. 79, n. 2, p. I 41-8, 2003.

I I. RUBELLO, D. et al. Natural course of subclinical hypothyroidism in Down syndrome: prospective study results and therapeutic considerations.J End Invest, v. 17, p. 35-40, 1995.

12. SELIKOWITZ, M. A five-year longitudinal study of thyroid function in children with Down syndrome. Dev Med Child Neurol, v. 35, n. 5, p. 396-40I, 1993.

13. SHALITIN, S.; PHILIP, M. Autoimmune thyroiditis in infants with Down's syndrome. J Ped End Metab, v. 15, n. 5, p. 649-52, 2002.

14. TUYSUZ, B.; BEKER, D. B. Thyroid dysfunction in children with Down's syndrome. Acta Paed, v. 90, n. I2, p. I389-93, 2001.

I5. ZORI, R. T. et al. Relationship of autoimmunity to thyroid dysfunction in children and adults with Down syndrome. Am J Med Gen Suppl, v. 7, p. 238-4I, 1990.

\section{Endereço para correspondência}

Renato Mitsunori Nisihara Laboratório de Imunopatologia Departamento de Patologia Médica/UFPR R. Padre Camargo, 280 - Curitiba-PR Tel.: (41) 360-1800, ramal 6.537 e-mail:renatomitsu@yahoo.com.br 